\title{
The role of the van der Waals interactions in the adsorption of anthracene and pentacene on the $\mathrm{Ag}(\mathbf{1 1 1})$ surface
}

\author{
Juliana M. Morbec ${ }^{1, \text { a) }}$ and Peter Kratzer ${ }^{1}$ \\ Faculty of Physics, University of Duisburg-Essen, Lotharstrasse 1, 47057 Duisburg, \\ Germany
}

Using first-principles calculations based on density-functional theory (DFT) we investigated the effects of the van der Waals (vdW) interactions on the structural and electronic properties of anthracene and pentacene adsorbed on the $\operatorname{Ag}(111)$ surface. We found that the inclusion of vdW corrections strongly affects the binding of both anthracene/ $\operatorname{Ag}(111)$ and pentacene/ $\operatorname{Ag}(111)$, yielding adsorption heights and energies more consistent with the experimental results than standard DFT calculations with generalized gradient approximation (GGA). For anthracene/Ag(111) the effect of the vdW interactions is even more dramatic: we found that "pure" DFT-GGA calculations (without including vdW corrections) result in preference for a tilted configuration, in contrast to experimental observations of flat-lying adsorption; including vdW corrections, on the other hand, alters the binding geometry of anthracene/ $\mathrm{Ag}(111)$, favoring the flat configuration. The electronic structure obtained using a self-consistent vdW scheme was found to be nearly indistinguishable from the conventional DFT electronic structure once the correct vdW geometry is employed for these physisorbed systems. Moreover, we show that a vdW correction scheme based on a hybrid functional DFT calculation (HSE) results in an improved description of the highest occupied molecular level of the adsorbed molecules.

\section{INTRODUCTION}

Interfaces between organic molecules and metal surfaces are fundamental constituents of numerous organic optoelectronic devices and play important roles in their performance. Thus, understanding the adsorption of organic molecules on metal surfaces and the interactions at such organic/metal interfaces is crucial to develop efficient devices.

Anthracene $\left(\mathrm{C}_{14} \mathrm{H}_{10}\right)$ and pentacene $\left(\mathrm{C}_{22} \mathrm{H}_{14}\right)$ adsorbed on the $\mathrm{Ag}(111)$ surface are particularly interesting systems because of the frequent use of silver as electrode and the promising properties of the anthracene and pentacene compounds; both compounds exhibit high carrier mobility ${ }^{1}$ and excellent electroluminescence and photoluminescence ${ }^{2}$, and have great potential for use in organic light-emitting diodes ${ }^{3}$ and organic field effect transistors $^{4}$. Experimental studies have reported that both anthracene and pentacene monolayers weakly adsorb on $\mathrm{Ag}(111)$, as characteristic of physisorption or weak chemisorption ${ }^{5-10}$, which suggests that van der Waals interactions (vdW) play an important role in the binding of these systems.

Van der Waals interactions are weak interactions that originate from non-local correlations between electronic charge fluctuations. They are the dominant interactions in physisorbed and weakly chemisorbed systems and are known to strongly affect the stability and structure of organic/metal interfaces ${ }^{11-14}$. Therefore, various schemes have been devised how to include vdW interactions into density functional theory (DFT). While some are formulated directly as functionals of the electron density ${ }^{15,16}$, the more common and convenient schemes simply add

\footnotetext{
a) Electronic mail: jmmorbec@gmail.com
}

a correction to the DFT total energy in the form of a pairwise interaction potential ${ }^{17,18}$.

In this work, we present a first-principles study on the effects of the vdW interactions on the structural and electronic properties of anthracene and pentacene adsorbed on the $\operatorname{Ag}(111)$ surface. We used both the vdW ${ }^{\text {surf }}$ approach ${ }^{19}$ and the many-body dispersion (MBD) method ${ }^{20,21}$ to treat the vdW interactions in our calculations. While the first works with an additive pairwise potential, albeit with density-dependent coefficient, the second approach is able to take cooperative effects into account. Both the vdW ${ }^{\text {surf }}$ and MBD methods have been shown to provide reliable adsorption energy and height for interfaces between organic molecules (such as benzene and PTCDA) and coinage metal surfaces ${ }^{22-24}$. Our results show that the inclusion of vdW corrections is crucial to correctly describe the flat adsorption geometry of anthracene on $\mathrm{Ag}(111)$; calculations using "pure" generalized gradient approximation (GGA), without including vdW corrections, resulted in a tilted configuration for anthracene $\operatorname{Ag}(111)$, while the use of the vdW $W^{\text {surf }}$ approach yielded flat molecular orientation, consistent with experimental observations. We also found that the adsorption heights and the adsorption energies in both anthracene/ $\mathrm{Ag}(111)$ and pentacene/ $\mathrm{Ag}(111)$ are strongly affected by the vdW treatment. On the other hand, we noticed that including the density-dependence inherent in the vdW ${ }^{\text {surf }}$ approach has only tiny effects on the electronic structure of the systems. Lastly, we computed the change in the work function of the $\mathrm{Ag}(111)$ surface upon the adsorption of anthracene and pentacene; we found that anthracene induces a larger reduction in the work function than pentacene, consistent with the stronger physisorption character observed in anthracene/ $\mathrm{Ag}(111)$ in comparison with pentacene/ $\mathrm{Ag}(111)$.

The rest of the paper is organized as follows: in Sec. II we describe the details of our first-principles calculations; 
in Sec. III we present and discuss our results for the structural and electronic properties of anthracene/ $\mathrm{Ag}(111)$ and pentacene $/ \operatorname{Ag}(111)$; and in Sec. IV we provide our conclusions.

\section{COMPUTATIONAL DETAILS}

All calculations were performed in the framework of the density-functional theory ${ }^{25}$ as implemented in the FHI-aims ${ }^{26}$, an all-electron code that uses numeric atomcentered orbitals as basis functions. We used the generalized gradient approximation (GGA) proposed by Perdew, Burke, and Ernzerhof (PBE) ${ }^{27}$ for the exchangecorrelation functional, as well as, the hybrid HeydScuseria-Ernzerhof (HSE06) ${ }^{28}$ functional. "Tight" settings from the FHI-aims code were used in all calculations, with "tier2" / "tier3" / "tier2" basis sets for $\mathrm{Ag} / \mathrm{C} / \mathrm{H}$ in the PBE calculations and "tier1" / "tier2" / "tier2" basis sets for $\mathrm{Ag} / \mathrm{C} / \mathrm{H}$ in the calculations with the HSE functional. Convergence criteria of $10^{-5}$ electrons $/ \AA^{3}$ and $10^{-5} \mathrm{eV}$ were applied for the charge density and the total energy, respectively.

To treat the vdW interactions we employed two approaches coupled to $\mathrm{PBE}$ and HSE functionals: (i) the $\mathrm{vdW}^{\text {surf }}$ approach ${ }^{19}$, which includes the collective electronic response of the substrate in the determination of the $\mathrm{vdW}$ parameters $\left(C_{6}\right.$ coefficients, polarizabilities and vdW radii) by combining the pairwise Tkatchenko-Scheffler (TS) method ${ }^{18}$ with the LifshitzZaremba-Kohn theory ${ }^{29,30}$ for the vdW interaction between an atom and a solid surface, and (ii) the manybody dispersion (MBD) method ${ }^{20,21}$, in which the atomic response functions are represented by a set of quantum harmonic oscillators and the screened long-range many-body vdW energy is computed using the adiabatic connection fluctuation-dissipation theorem within the dipole approximation. In the calculations with the $\mathrm{vdW}^{\text {surf }}$ approach we used the screened vdW parameters computed in Ref. 19. Throughout the paper we will refer to the calculations without including $\mathrm{vdW}$ corrections as "PBE calculations" (or "HSE calculations", when the hybrid HSE functional is used), and we will use "PBE(HSE)+vdW"surf" and "PBE(HSE)+MBD" for the calculations with the $\mathrm{vdW}^{\text {surf }}$ and MBD approaches, respectively. To examine the effects of the $\mathrm{vdW}$ interactions on the electronic properties of anthracene $/ \operatorname{Ag}(111)$ and pentacene $/ \operatorname{Ag}(111)$, we used the self-consistent $\mathrm{vdW}^{\text {surf }}$ (sc-vdW $\left.{ }^{\text {surf }}\right)$ method $^{31}$, in which the functional derivative (with respect to the density) of the pairwise TS vdW potential is added to the exchangecorrelation potential to form the total effective KohnSham potential.

In order to build the supercells for our calculations, we first computed the lattice constant of bulk silver; using a $16 \times 16 \times 16$ Monkhorst-Pack $k$-point mesh, we found $a=4.140 \AA$ and $a=4.020 \AA$ in the PBE and $\mathrm{PBE}+\mathrm{vdW} \mathrm{W}^{\text {surf }}$ calculations, respectively, which are in close agreement with the experimental value of $4.09 \AA^{32}$ and with previous theoretical results ${ }^{33}$ obtained using PBE $(4.149 \AA)$ and $\mathrm{PBE}+\mathrm{vdW}^{\text {surf }}(4.007 \AA)$. We used the respective computed lattice constants in our PBE and $\mathrm{PBE}+\mathrm{vdW}{ }^{\text {surf }}$ calculations.

Anthracene $/ \operatorname{Ag}(111)$ was modeled using a $(2 \sqrt{3} \times$ $2 \sqrt{3}) R 30^{\circ}$ surface unit cell (in accordance with the STM measurements of anthracene adlayers on $\mathrm{Ag}$ surfaces in perchloric acid solution reported by Shimooka et al. ${ }^{6}$ ) with a five-layer slab; for pentacene/ $\operatorname{Ag}(111)$ we used a $(6 \times 3)$ surface unit cell (which is consistent with experimental measurements ${ }^{9}$ ) with a four-layer slab. A vacuum region of $\sim 30 \AA$ was used in both cases to avoid unphysical interaction between periodic images. In the geometry optimizations, the molecules and the top two silver layers were allowed to relax while the remaining bottom layers were constrained to their bulk positions; a force convergence criterion of $10^{-2} \mathrm{eV} / \AA$ was applied for structural relaxations. We used $4 \times 4 \times 1$ and $3 \times 6 \times 1$ Monkhorst-Pack $k$-point meshes in the structure optimization and total-energy calculations of anthracene $/ \mathrm{Ag}(111)$ and pentacene $/ \mathrm{Ag}(111)$ systems, respectively, whilst denser $k$-point sets, $12 \times 12 \times 1$ for anthracene $/ \operatorname{Ag}(111)$ and $9 \times 18 \times 1$ for pentacene/ $\operatorname{Ag}(111)$, were employed to compute the density of states and the work functions. For the calculations of the work function we examined the convergence of our results with respect to the number of layers and the thickness of the vacuum region.

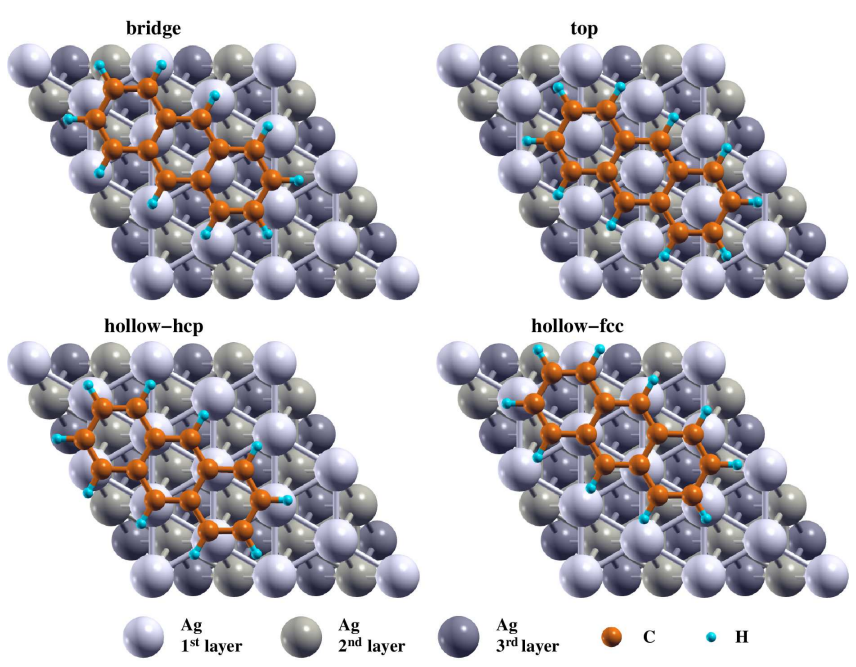

FIG. 1. Ball and stick representation of the adsorption sites (bridge, top, hollow-hcp, and hollow-fcc) examined for anthracene/Ag(111). 


\section{RESULTS AND DISCUSSION}

\section{A. Anthracene on $\mathrm{Ag}(\mathbf{1 1 1})$}

We first determined the equilibrium geometry for anthracene/ $\mathrm{Ag}(111)$. Considering only planar adsorption and taking as reference the central carbon ring, we examined four adsorption sites (see Fig. 1): bridge, where the central ring lies over a $\mathrm{Ag}-\mathrm{Ag}$ bond; top, where the central ring is on top of a $\mathrm{Ag}$ atom from the top layer; hollow-hcp, where the central ring is on top of a 2nd-layer Ag atom; and hollow-fcc, where the central ring is on top of a 3rd-layer Ag atom. The most stable adsorption site was found to be hollow-hcp in both $\mathrm{PBE}$ and $\mathrm{PBE}+\mathrm{vdW} \mathrm{Wurf}^{\text {surf }}$ calculations (see Table I), with total energy 1-4 meV smaller than the second most stable configuration, hollow-fcc. We found, however, that PBE calculations favored a tilted hcp-configuration (Fig. 2) with the molecular short axis making a tilt angle of $\sim 26.7^{\circ}$ with the substrate, in contrast to previous experimental observations ${ }^{5-7}$ of flat adsorption of anthracene molecules on the $\mathrm{Ag}(111)$ surface. We found that the flat hollow-hcp configuration is $0.098 \mathrm{eV}$ less stable than the tilted configuration, and it has adsorption height of $4.095 \AA$ and adsorption energy of $-0.061 \mathrm{eV}$.

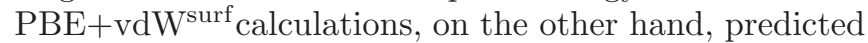
flat geometry (see Fig. 2) with adsorption energy of $-1.380 \mathrm{eV}$ (see Table I), more than $1.3 \mathrm{eV}$ stronger than that predicted by PBE calculations, and adsorption height of $3.015 \AA$, significantly smaller than the adsorption height obtained without including vdW corrections $(4.432 \AA)$ and in the range of typical moleculesubstrate distances for aromatic molecules on metals $(2.8-3.2 \AA)^{34,35}$. The adsorption height $d$ is calculated as the vertical distance between the molecule's center of mass and the average positions of the $\mathrm{Ag}$ atoms in the uppermost layer. In the hcp configuration, the surfacelayer $\mathrm{Ag}$ atoms below the anthracene molecule relaxed inward by $\sim 0.02 \AA$ on average. The adsorption energy $E_{\text {ads }}$ is computed using

$$
E_{\mathrm{ads}}=E_{\mathrm{Mol} / \mathrm{Ag}(111)}-E_{\mathrm{Ag}(111)}-E_{\mathrm{Mol}},
$$

where $E_{\mathrm{Mol} / \mathrm{Ag}(111)}, E_{\mathrm{Ag}(111)}$ and $E_{\mathrm{Mol}}$ are the total energies of the adsorbed system, the bare $\mathrm{Ag}(111)$ surface, and the single molecule in a large supercell, respectively.
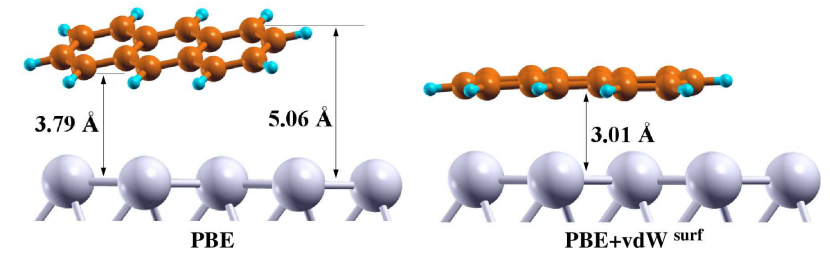

FIG. 2. Equilibrium geometries of anthracene/ $\mathrm{Ag}(111)$ (hollow-hcp configuration) obtained using the PBE (left) and $\mathrm{PBE}+\mathrm{vdW} \mathrm{W}^{\text {surf }}$ (right) methods.
TABLE I. Relative total energies $\Delta E_{T}$ (in eV), adsorption energies $E_{\text {ads }}$ (in $\mathrm{eV}$ ), and adsorption heights $d$ (in $\AA$ ) for anthracene $/ \operatorname{Ag}(111)$ at different adsorption sites, computed using the PBE and PBE+vdW ${ }^{\text {surf }}$ methods. The equilibrium orientation of the molecule with respect to the substrate (flat or tilted) is indicated within brackets. $\Delta E_{T}$ is calculated as the difference in total energy of a given configuration and the most stable configuration (hcp). The adsorption height is determined as the vertical distance between the center of mass of the molecule and the average positions of the Ag atoms in the topmost layer. The values in parentheses in column 6 are the adsorption energies obtained with the PBE+MBD method at the PBE+vdW ${ }^{\text {surf }}$ geometry.

\begin{tabular}{|c|c|c|c|c|c|c|}
\hline \multirow[b]{2}{*}{ site } & \multicolumn{3}{|c|}{ PBE } & \multicolumn{3}{|c|}{$\mathrm{PBE}+\mathrm{vdW} \mathrm{W}^{\text {surf }}$} \\
\hline & $\Delta E_{T}$ & $E_{\text {ads }}$ & $d$ & $\Delta E_{T}$ & $E_{\text {ads }}$ & $d$ \\
\hline bridge & 0.106 & -0.060 & $\begin{array}{l}4.026 \\
\text { [flat] }\end{array}$ & 0.074 & $\begin{array}{c}-1.313 \\
(-0.856)\end{array}$ & $\begin{array}{l}3.036 \\
\text { [flat] }\end{array}$ \\
\hline top & 0.111 & 7 & $\begin{array}{l}4.112 \\
\text { [flat] }\end{array}$ & 0.155 & $\begin{array}{c}-1.255 \\
(-0.763)\end{array}$ & $\begin{array}{l}3.047 \\
\text { [flat] }\end{array}$ \\
\hline hcp & 0.000 & -0.065 & $\begin{array}{c}4.432 \\
\text { [tilted] }\end{array}$ & 0.000 & $\begin{array}{l}-1.380 \\
(-0.925)\end{array}$ & $\begin{array}{l}3.015 \\
\text { [flat] }\end{array}$ \\
\hline$f_{c c}$ & 0.001 & -0.064 & $\begin{array}{c}4.440 \\
\text { [tilted] }\end{array}$ & 0.004 & $\begin{array}{c}-1.363 \\
(-0.891)\end{array}$ & $\begin{array}{l}3.017 \\
\text { [flat] }\end{array}$ \\
\hline
\end{tabular}

The failure of $\mathrm{PBE}$ to describe the interaction and structural properties of anthracene/ $\mathrm{Ag}(111)$ can also be seen in Fig. 3(a), which shows the adsorption energy $E_{\text {ads }}$ of hcp-anthracene $/ \operatorname{Ag}(111)$, computed using different approaches, as a function of the adsorption height $d$; the geometry of the system was constrained to the PBE equilibrium geometry in the PBE calculations and to the $\mathrm{PBE}+\mathrm{vdW}^{\text {surf }}$ geometry in the calculations with the $\mathrm{PBE}+\mathrm{vdW} \mathrm{W}^{\text {surf }}$ and $\mathrm{PBE}+\mathrm{MBD}$ methods, and only the adsorption height was changed. "Pure" PBE calculations, without inclusion of $\mathrm{vdW}$ corrections, predicted repulsive interaction for distances smaller than $4 \AA$, and very small attractive adsorption energies $(<0.07 \mathrm{eV})$ for larger distances, suggesting that the molecule does not bind to the surface. The inclusion of vdW corrections, on the other hand, pulled the molecule closer to the surface, bringing the adsorption height to values between 3.0 and $3.1 \AA$ (Fig. 3(a)); the curves obtained using the $\mathrm{PBE}+\mathrm{vdW}{ }^{\text {surf }}$ and $\mathrm{PBE}+\mathrm{MBD}$ methods exhibit pronounced minima of about -1.4 and $-0.9 \mathrm{eV}$, respectively. To the best of our knowledge, the adsorption height and energy of anthracene/ $\mathrm{Ag}(111)$ have not yet been determined experimentally. Nevertheless, we note that our adsorption energies computed using the $\mathrm{PBE}+\mathrm{vdW}^{\text {surf }}\left(E_{\text {ads }}=-1.380 \mathrm{eV}\right)$ and $\mathrm{PBE}+\mathrm{MBD}$ $\left(E_{\mathrm{ads}}=-0.943 \mathrm{eV}\right)$ methods are within the range of experimental values reported for benzene $/ \mathrm{Ag}(111), 0.68 \pm$ $0.05 \mathrm{eV}^{36,37}$, naphthalene/ $\mathrm{Ag}(111), 1.03 \pm 0.05 \mathrm{eV}^{37,38}$, and pentacene $/ \mathrm{Ag}(111), 1.5 \mathrm{eV}^{9}$. The adsorption height obtained using the PBE+vdW ${ }^{\text {surf }}$ method, $3.015 \AA$, is also close to that reported for benzene/ $\mathrm{Ag}(111), 3.04 \pm$ 

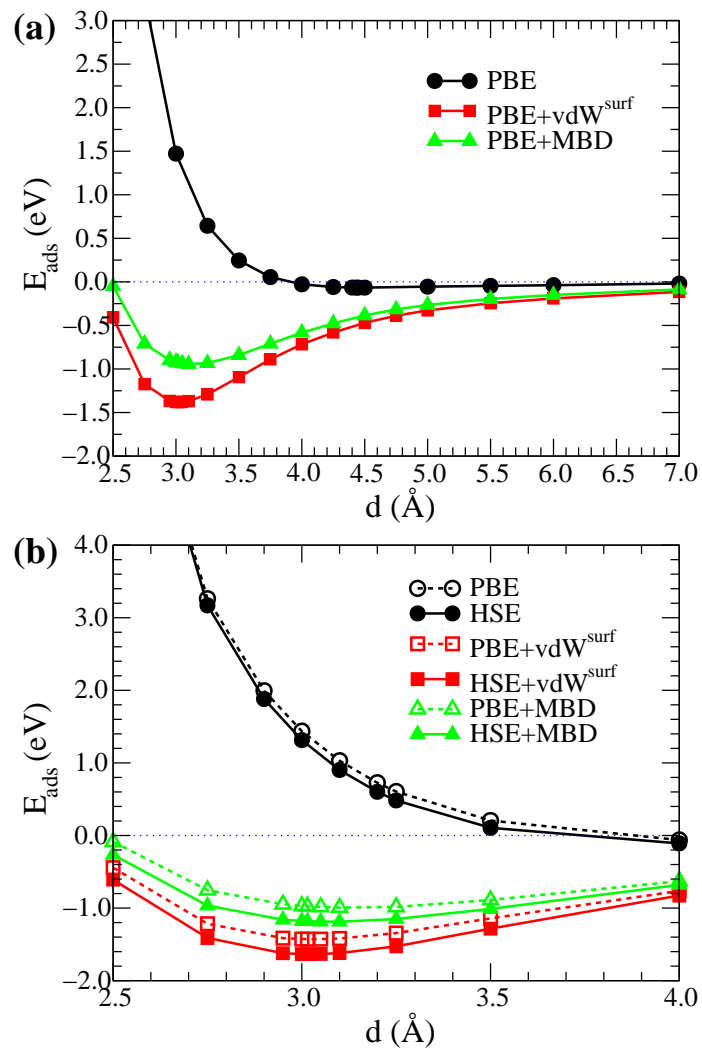

FIG. 3. Adsorption energy $E_{\text {ads }}$ of hcp-anthracene/ $\operatorname{Ag}(111)$ as a function of the adsorption height $d$, computed using the (a) $\mathrm{PBE}, \mathrm{PBE}+\mathrm{vdW} \mathrm{surf}^{\text {sun }} \mathrm{PBE}+\mathrm{MBD}$, and (b) HSE, HSE+vdW ${ }^{\text {surf }}$ and HSE+MBD approaches. The geometry of the system was constrained to the PBE equilibrium geometry in the $\mathrm{PBE} / \mathrm{HSE}$ calculations and to

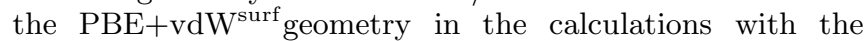
$\mathrm{PBE} / \mathrm{HSE}+\mathrm{vdW} \mathrm{W}^{\text {surf }}$ and $\mathrm{PBE} / \mathrm{HSE}+\mathrm{MBD}$ methods, and only the adsorption height was changed.

$0.02 \AA^{36}$. Previous theoretical studies ${ }^{22,36}$ have shown that the $\mathrm{vdW}^{\text {surf }}$ approach overestimates the adsorption energy of atoms and molecules adsorbed on metal surfaces, while the MBD method, which goes beyond the pairwise method and includes many-body dispersion effects, predicts results in better agreement with experiments; therefore, we expect that our PBE+MBD calculations would yield more accurate values for the adsorption energy and height of realistic anthracene/ $\mathrm{Ag}(111)$ systems.

We also examined the effects of including part of the short-range exact exchange, by using the HSE06 functional, on the adsorption energy of anthracene/ $\mathrm{Ag}(111)$. As can be seen in Fig. 3(b), the reduction of the selfinteraction error leads to an increase of about $0.2 \mathrm{eV}$ in the absolute values of the adsorption energy, with both the $\mathrm{vdW}^{\text {surf }}$ and MBD methods. The same behavior was recently observed for benzene $/ \operatorname{Ag}(111)^{36}$; in this case, the adsorption energy obtained with HSE+MBD was in better agreement with experimental results than that obtained with the PBE+MBD method. In our case, for an- thracene $/ \operatorname{Ag}(111)$, the adsorption energy obtained using the HSE+MBD method $\left(E_{\mathrm{ads}}=-1.18 \mathrm{eV}\right)$ is still within range of the experimental values reported for other oligoacenes, such as benzene, naphthalene and pentacene, adsorbed on the $\mathrm{Ag}(111)$ surface $(0.68-1.5 \mathrm{eV})^{9,36-38}$.

In addition to the structural properties, we also investigated the electronic properties of anthracene $/ \mathrm{Ag}(111)$. Figure 4 shows the density of states (DOS) of the isolated anthracene (Fig. 4(a)) and the adsorbed molecule in the PBE+vdW ${ }^{\text {surf }}$ optimized geometry (Figs. 4(b)-(d)); for comparison, the DOS of the adsorbed molecule in the PBE geometry is shown in Fig. S1 of the Supplemental Material. For the adsorbed system in the $\mathrm{PBE}+\mathrm{vdW}^{\text {surf }}$ geometry we present the DOS obtained without including vdW interactions ("pure" PBE and HSE calculations, shown in Figs. 4(b) and (c), respectively) and with vdW corrections using the self-consistent vdW $^{\text {surf }}\left(\right.$ sc-vdW $\left.{ }^{\text {surf }}\right)$ method $^{31}$ (Fig. 4(d)). The highest occupied (HOMO) and lowest unoccupied (LUMO) orbitals are formed by bonding $\pi$ and antibonding $\pi^{*}$ superpositions of the carbon $p_{z}$ orbitals above and below the molecular plane. Comparing the DOS of the isolated (Fig. 4(a)) and adsorbed (Fig. 4(b)) anthracene, we found that the presence of the substrate induced only a small decrease in the HOMO-LUMO gap $(\sim 0.13 \mathrm{eV})$ and a slight broadening in the HOMO and LUMO peaks (see also Fig. S2 of the Supplemental Material). On the other hand, the states located between 2.5 and $7 \mathrm{eV}$ below the Fermi level are strongly affected the hybridization between the molecular orbitals (mainly composed of $\mathrm{C} p$ orbitals (Fig. S2)) and the $\mathrm{Ag} d$ states, which are the dominant states between 2.5 and $7 \mathrm{eV}$ (Fig. 4(e)). Figs. 4(b) and (c) also show that both PBE and HSE calculations predicted that the LUMO remains unoccupied on the $\operatorname{Ag}(111)$ surface, indicating that there is no charge transfer between the substrate and the molecule and that anthracene is physisorbed on $\operatorname{Ag}(111)$, which is consistent with previous experimental studies ${ }^{5,7}$.

As expected, the PBE functional significantly underestimates the HOMO-LUMO band gap of the molecule; for the isolated molecule (Fig. 4(a)) we found a HOMOLUMO gap of $2.31 \mathrm{eV}$, in good agreement with previous theoretical result $(2.25 \mathrm{eV})^{39}$ but $\sim 66 \%$ underestimated with respect to the experimental value $(6.9 \mathrm{eV})^{39}$. The HSE hybrid functional brings the HOMO to lower energies, yielding a larger band gap, $2.97 \mathrm{eV}$ (still 57\% smaller than the experimental value). HSE also improves the prediction of the HOMO position of the adsorbed molecule; while with the PBE functional we obtained HOMO located at $1.81 \mathrm{eV}$ below the Fermi level (Fig. 4(b)), our HSE DOS (Fig. 4(c)) shows a pronounced peak at $2.33 \mathrm{eV}$ below the Fermi level, which is in good agreement with the experimental observation of a $\pi$-character band at the top of the valence band located at $2.5 \mathrm{eV}$ below the Fermi edge ${ }^{5}$. Both PBE and HSE functionals, however, fail to describe the position of the LUMO; we found that the LUMO is located right above the Fermi level $(0.37 \mathrm{eV}$ from PBE 
(a)

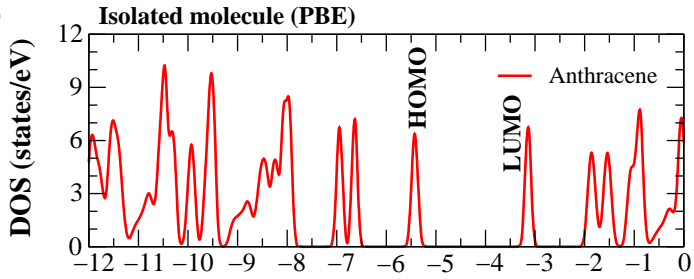

(b)

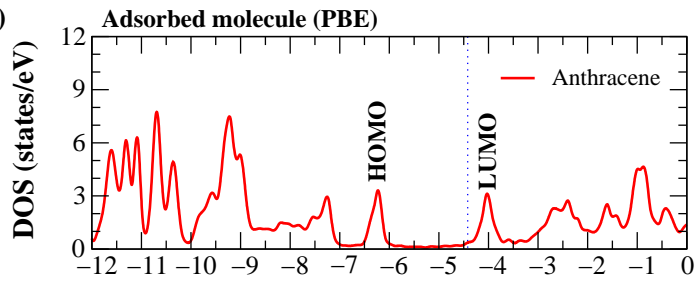

(c)

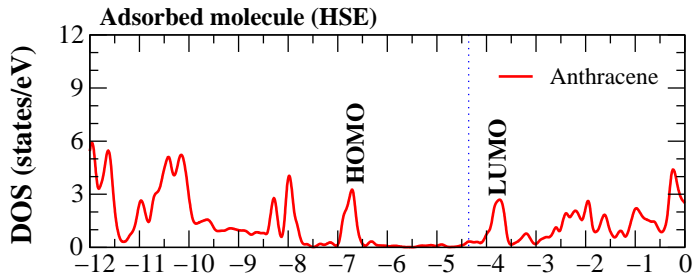

(d)

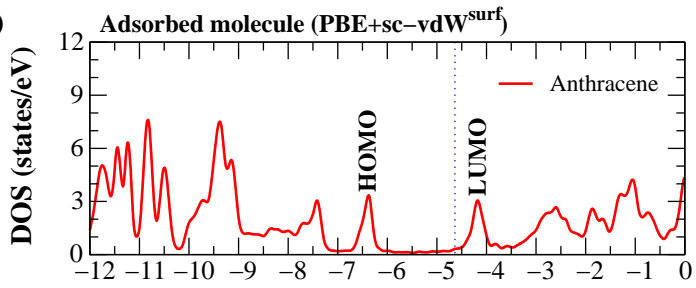

(e)

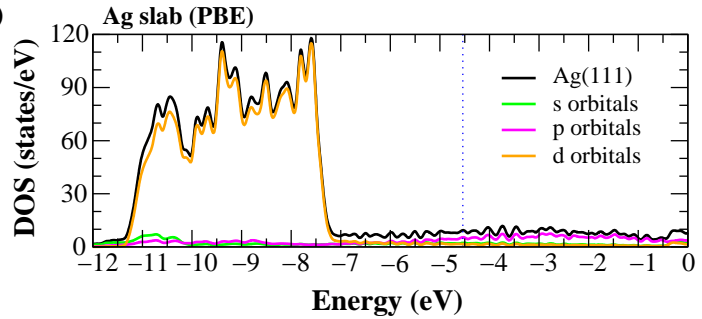

FIG. 4. Calculated density of states (DOS) of the anthracene $/ \operatorname{Ag}(111)$ system in the optimized $\mathrm{PBE}+\mathrm{vdW} \mathrm{W}^{\text {surf }}$ geometry. (a) Isolated anthracene, (bd) density of states projected onto the molecular orbitals of anthracene $/ \operatorname{Ag}(111)$ obtained with (b) PBE and (c) HSE functionals and (d) the $\mathrm{PBE}+\mathrm{sc}-\mathrm{vdW} \mathrm{W}^{\text {surf }}$ method, and (e) clean $\operatorname{Ag}(111)$ surface. The zero on the energy scale corresponds to the vacuum level. The dashed lines indicate the position of the Fermi level.

and $0.64 \mathrm{eV}$ from HSE calculation) whereas inverse photoemission measurements ${ }^{5}$ have shown that the LUMO is located between 2 and $3 \mathrm{eV}$ above the Fermi level. This underestimation is mainly due to the inability of KohnSham energy levels to describe excited states.

We examined the effect of including vdW corrections in the description of the electronic structure of anthracene $/ \mathrm{Ag}(111)$, after the correct geometry has been obtained. Thus, considering the anthracene/ $\mathrm{Ag}(111)$ sys-

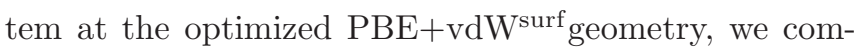
pare the DOS of the adsorbed molecule obtained using the PBE functional without vdW corrections (Fig. 4(b)) with that obtained using the $\mathrm{PBE}+\mathrm{sc}-\mathrm{vdW} \mathrm{W}^{\text {surf }}$ method (Fig. 4(d)); we found that the inclusion of vdW corrections has, apart from a shift of the Fermi level, no significant effect on the electronic properties of the system (see also Fig. S3 of the Supplemental Material). Our results therefore show that, although a proper treatment of the vdW interactions is crucial to correctly describe the stability and structural properties of anthracene $/ \mathrm{Ag}(111)$, vdW interactions have little effects on the electronic structure of the system once the correct geometry has been obtained. The effect of the vdW interactions on the electronic structure is therefore indirect, via the correct description of the geometry of the system; the DOS of the adsorbed molecule at the PBE geometry (see Fig. S1(a)) is similar to the DOS of the isolated molecule (Fig. 4(a)), while the DOS of the adsorbed molecule at the $\mathrm{PBE}+\mathrm{vdW}^{\text {surf }}$ geometry (Fig. 4(b)) is broader with the LUMO state located closer to the Fermi level (see also Fig. S1 for a comparison between the DOS of the adsorbed molecule at the PBE and $\mathrm{PBE}+\mathrm{vdW}{ }^{\text {surf }}$ geometries). We note that by electronic structure we mean the relative position of the bands with respect to each other and the Fermi level; a shift of the energetic reference point, i.e. the vacuum energy, is much more difficult to detect from a plot of the DOS.

The redistribution of charge upon adsorption is most sensitively reflected in a change of the surface dipole, and hence in the work function. Therefore, we computed the change in the work function of the $\mathrm{Ag}(111)$ surface due to the adsorption of an anthracene monolayer. All calculations were performed using the $\mathrm{PBE}+\mathrm{vdW} \mathrm{Wurf}^{\text {suptimized }}$ geometry. For the work function of the clean $\mathrm{Ag}(111)$ surface we obtained $4.43 \mathrm{eV}$ without including $\mathrm{vdW}$ corrections ("pure" PBE calculations) and $4.81 \mathrm{eV}$ with the PBE+sc-vdW $W^{\text {surf }}$ method, which are in good agreement with previous theoretical results $\left(4.44^{31}\right.$ and $4.45 \mathrm{eV}^{40}$ with $\mathrm{PBE}$ and $4.74 \mathrm{eV}^{31}$ with the PBE+sc$\mathrm{vdW}^{\text {surf }}$ approach) and with experimental values $(4.45-$ $\left.4.90 \mathrm{eV}^{7,8,41-43}\right)$. The higher work function in the $\mathrm{vdW}^{\text {surf }}$ calculations indicates that the electronic charge density extends somewhat further outside the surface. As shown and discussed by Ferri et al. in Ref. 31, the inclusion of long-range correlation effects in the self-consistent electronic structure calculation leads to a net accumulation of the charge density above the surface and between the metal layers and to a depletion at the top metal layers, compared to a PBE calculation with vdW interactions treated by pairwise potentials. This lowers the Fermi level (due to the Coulomb attraction between the metal layers) and shifts the vacuum level to higher values (due to the extension of the charge density above the surface), consequently increasing the work function. Our calculations show that the adsorption of anthracene on $\mathrm{Ag}(111)$ leads to a significant reduction in the work function of the surface; using the PBE + sc- 


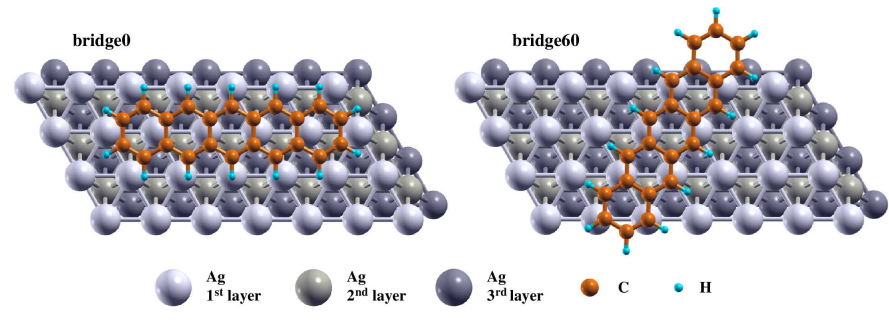

FIG. 5. Ball and stick representation of the bridge0 and bridge 60 configurations for pentacene/ $\mathrm{Ag}(111)$.

${ }_{\mathrm{vdW}}{ }^{\text {surf }}$ method we found $3.77 \mathrm{eV}$ for the work function of anthracene/ $\mathrm{Ag}(111)$, which means a reduction of $1.04 \mathrm{eV}$ with respect to the clean surface, while our "pure" PBE calculations yielded a decrease of $0.81 \mathrm{eV}$ (from 4.43 to $3.62 \mathrm{eV}$ ). The large reduction in the work function is due to the push-back effect arising from the Pauli repulsion between the electron density of the molecule and the surface electrons, which is dominant in physisorbed systems. We notice that our PBE and PBE+sc-vdW ${ }^{\text {surf }}$ results for the change in the work function $(-0.81$ and $-1.04 \mathrm{eV}$, respectively) are significantly larger than experimental values obtained by measuring the onset current into the sample, $-0.5 \pm 0.1 \mathrm{eV}^{5}$, and by determining the photoemission kinetic energy width, $-0.68 \pm 0.02 \mathrm{eV}^{44}$. It should be mentioned, however, that the experimental data available were obtained for multilayer anthracene adsorption and/or for lower molecular density. We also estimated the change in the positions of the anthracene HOMO and LUMO states with respect to the vacuum level upon adsorption: from PBE calculations we obtained shifts of 0.80 and $0.92 \mathrm{eV}$ towards lower energies for $\mathrm{HOMO}$ and LUMO, respectively, while PBE+sc-vdW ${ }^{\text {surf }}$ yielded shifts of 0.92 and $1.06 \mathrm{eV}$. Previous angle-resolved photoemission and inverse photoemission measurements predicted rigid shifts to lower energies of about $0.9 \mathrm{eV}$ for the occupied states and $1.1 \mathrm{eV}$ for the unoccupied orbitals ${ }^{5}$.

\section{B. Pentacene on $\mathrm{Ag}(111)$}

Pentacene monolayers have been experimentally observed to adsorb parallel to the $\mathrm{Ag}(111)$ surface $^{10,45,46}$; therefore, in this work we considered only planar adsorption for pentacene/ $\mathrm{Ag}(111)$. We examined eight adsorption sites: four configurations (bridge0, top0, hcp0, and fcc0) in which the molecular long axis is parallel to the larger vector of the surface unit cell and four configurations (bridge60, top60, hcp60, and fcc60) in which the molecular long axis is rotate $60^{\circ}$ with respect to the former configurations. Figure 5 shows a schematic top view of the configurations bridge 0 and bridge 60 .

We optimized all configurations using the PBE and PBE+vdW ${ }^{\text {surf }}$ methods and we found, with both methods, that bridge60 is the most favorable configuration (see Table II). The differences in total energy between bridge60 and the other configurations obtained using the PBE+vdW ${ }^{\text {surf }}$ method (15-226 meV) are significantly larger than those obtained in the "pure" PBE calculations $(1-24 \mathrm{meV})$. This is mainly due to the smaller adsorption heights predicted by the $\mathrm{PBE}+\mathrm{vd} \mathrm{W}^{\text {surf }}$ method: as can be seen in Table II, PBE+vdW ${ }^{\text {surf }}$ method yielded adsorption heights between 2.8 and $3.0 \AA$, while the PBE values are larger than $3.9 \AA$. The small difference in total energy between the configurations bridge60, hcp60, and hcp0 ( $\sim 15 \mathrm{meV}$ with the $\mathrm{PBE}+\mathrm{vd} \mathrm{W}^{\text {surf }}$ method) also suggests that different adsorption configurations may coexist in pentacene/ $\mathrm{Ag}(111)$, as observed in experiments $^{9}$. Both $\mathrm{PBE}$ and $\mathrm{PBE}+\mathrm{vdW} \mathrm{Wurf}^{\text {, how- }}$ ever, predict flat conformation for pentacene/Ag(111), as can be seen in Fig. 6, in agreement with experimental observations ${ }^{10,45,46}$. Our results are also in good agreement with previous theoretical studies: from PBE calculations we found $d=3.938 \AA$ and $E_{\text {ads }}=$ $-0.119 \mathrm{eV}$, which compare well with previous GGA results $\left(d=3.7-4.12 \AA\right.$ and $E_{\text {ads }}$ within the range of -0.078 to $-0.108 \mathrm{eV})^{47,48}$; using the $\mathrm{PBE}+\mathrm{vdW} \mathrm{Wurf}^{\text {sur }}$ method we found $d=2.910 \AA$ and $E_{\mathrm{ads}}=-2.396 \mathrm{eV}$, which is in good agreement with previous results obtained by Toyoda et al. ${ }^{47}$ using the pairwise DFT-D method $(d=2.9 \AA$ and $E_{\text {ads }}=-2.28 \mathrm{eV}$ ), but differ from the values obtained by the same authors using the nonlocal vdW-DF method $\left(d=3.7 \AA \text { and } E_{\text {ads }}=-1.62 \mathrm{eV}\right)^{47}$. It should be pointed out that the vdW-DF method tends to overestimate the adsorption heights, even though it provides reliable adsorption energies ${ }^{12,22}$ - recent vdW-DF functionals (e.g., optB86b-vdW and rev-vdW-DF2), however, have been shown to give both accurate adsorption height and energy ${ }^{49}$. As can be seen in Table II, the inclusion of many-body effects leads to a reduction of the adsorption energy to $E_{\text {ads }}=-1.652 \mathrm{eV}$, in close agreement to the vdW-DF value.

Figure 7(a) shows the adsorption energy of bridge60pentacene $/ \operatorname{Ag}(111)$ as a function of the adsorption height, computed using different methods. Similar to what was observed for anthracene/Ag(111), "pure" PBE calculations predicted low adsorption energies $(<$ $0.12 \mathrm{eV})$ and large adsorption heights $(\sim 3.9 \AA)$ suggesting that the molecule does not bind to the surface. The inclusion of the vdW interactions, again, brought the molecule closer to the surface $(\sim 2.9 \AA)$ and increased the absolute value of the adsorption energy. Using the $\mathrm{PBE}+\mathrm{MBD}$ method we found $E_{\mathrm{ads}}=-1.652 \mathrm{eV}$, in good agreement with both the theoretical result $(-1.62 \mathrm{eV})$ obtained by Toyoda et al. ${ }^{47}$, who used the vdW-DF method,

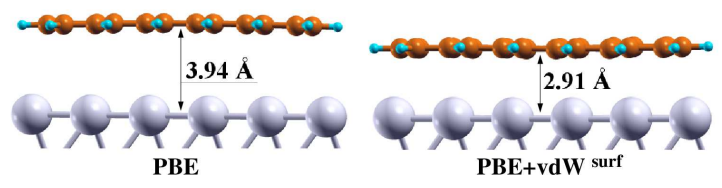

FIG. 6. Equilibrium geometries of pentacene/Ag(111) (bridge60 configuration) obtained using the PBE (left) and PBE+vdW ${ }^{\text {surf }}$ (right) methods. 
TABLE II. Relative total energies $\Delta E_{T}$ (in eV), adsorption energies $E_{\text {ads }}$ (in $\mathrm{eV}$ ), and adsorption heights $d$ (in $\AA$ ) for pentacene $/ \mathrm{Ag}(111)$ at different adsorption sites, computed using the PBE and PBE+vdW ${ }^{\text {surf }}$ methods. The equilibrium orientation of the molecule with respect to the substrate (flat or tilted) is indicated within brackets. $\Delta E_{T}$ is calculated as the difference in total energy of a given configuration and the most stable configuration (bridge60). The adsorption height is taken as the vertical distance between the center of mass of the molecule and the average positions of the $\mathrm{Ag}$ atoms in the topmost layer. The values in parentheses in column 6 are the adsorption energies obtained with the PBE+MBD method at the $\mathrm{PBE}+\mathrm{vdW}^{\text {surf }}$ geometry.

\begin{tabular}{|c|c|c|c|c|c|c|}
\hline \multirow[b]{2}{*}{ site } & \multicolumn{3}{|c|}{ PBE } & \multicolumn{3}{|c|}{$\mathrm{PBE}+\mathrm{vdW} \mathrm{W}^{\text {surf }}$} \\
\hline & $\Delta E_{T}$ & $E_{\text {ads }}$ & $d$ & $\Delta E_{T}$ & $E_{\text {ads }}$ & $d$ \\
\hline bridge0 & 0.017 & -0.102 & $\begin{array}{l}4.097 \\
\text { [flat] }\end{array}$ & 0.174 & $\begin{array}{c}-2.189 \\
(-1.400)\end{array}$ & $\begin{array}{l}2.956 \\
\text { [flat] }\end{array}$ \\
\hline top0 & 0.017 & -0.102 & $\begin{array}{l}4.081 \\
\text { [flat] }\end{array}$ & 0.226 & $\begin{array}{c}-2.119 \\
(-1.295)\end{array}$ & $\begin{array}{l}2.965 \\
\text { [flat] }\end{array}$ \\
\hline hcp0 & 0.002 & -0.117 & $\begin{array}{l}3.942 \\
\text { [flat] }\end{array}$ & 0.015 & $\begin{array}{c}-2.378 \\
(-1.646)\end{array}$ & $\begin{array}{l}2.902 \\
\text { [flat] }\end{array}$ \\
\hline fcc0 & 0.001 & -0.118 & $\begin{array}{c}3.943 \\
\text { [flat] }\end{array}$ & 0.052 & $\begin{array}{c}-2.331 \\
(-1.585)\end{array}$ & $\begin{array}{l}2.823 \\
\text { [flat] }\end{array}$ \\
\hline bridge60 & 0.000 & -0.119 & $\begin{array}{l}3.938 \\
\text { [flat] }\end{array}$ & 0.000 & $\begin{array}{c}-2.396 \\
(-1.652)\end{array}$ & $\begin{array}{l}2.910 \\
\text { [flat] }\end{array}$ \\
\hline top60 & 0.024 & -0.095 & $\begin{array}{l}3.946 \\
\text { [flat] }\end{array}$ & 0.226 & $\begin{array}{c}-2.120 \\
(-1.295)\end{array}$ & $\begin{array}{l}2.964 \\
\text { [flat] }\end{array}$ \\
\hline hсp60 & 0.011 & -0.111 & $\begin{array}{l}3.947 \\
\text { [flat] }\end{array}$ & 0.015 & $\begin{array}{c}-2.379 \\
(-1.646)\end{array}$ & $\begin{array}{l}2.901 \\
\text { [flat] }\end{array}$ \\
\hline fcc60 & 0.006 & -0.113 & $\begin{array}{c}3.943 \\
\text { [flat] }\end{array}$ & 0.034 & $\begin{array}{c}-2.336 \\
(-1.600)\end{array}$ & $\begin{array}{l}2.921 \\
\text { [flat] }\end{array}$ \\
\hline
\end{tabular}

and with the experimental value of the desorption energy of pentacene $/ \mathrm{Ag}(111), 1.5 \mathrm{eV}^{9}$. The comparison between our computed adsorption energies and the experimental value of the desorption energy shows that (i) the $\mathrm{PBE}+\mathrm{vdW} \mathrm{W}^{\text {surf }}$ method overestimates the adsorption energy, as has been observed for other systems ${ }^{22,36}$, and (ii) the inclusion of many-body effects is important to correctly describe the interaction between pentacene and the $\operatorname{Ag}(111)$ surface. Figure 7(b) shows that the hybrid HSE functional leads to an increase of $\sim 0.2 \mathrm{eV}$ in the absolute values of the adsorption energy, similar to what we found for anthracene $/ \operatorname{Ag}(111)$.

The density of states of bridge60-pentacene/ $\mathrm{Ag}(111)$ is displayed in Fig. 8. Since the $\pi$ and $\pi^{*}$ orbitals are more delocalized in pentacene compared to anthracene, the energy splitting between HOMO and LUMO is smaller. Similar to what was observed for anthracene, the presence of the substrate has small effects on the relative position of the HOMO and LUMO states (see Figs. 8(a) and (b) and Fig. S5 of the Supplemental Material). We found, however, that the LUMO level in pentacene $/ \operatorname{Ag}(111)$ is located closer to the Fermi level than in anthracene/ $\operatorname{Ag}(111)$. Our PBE (Fig. 8(b)) and HSE
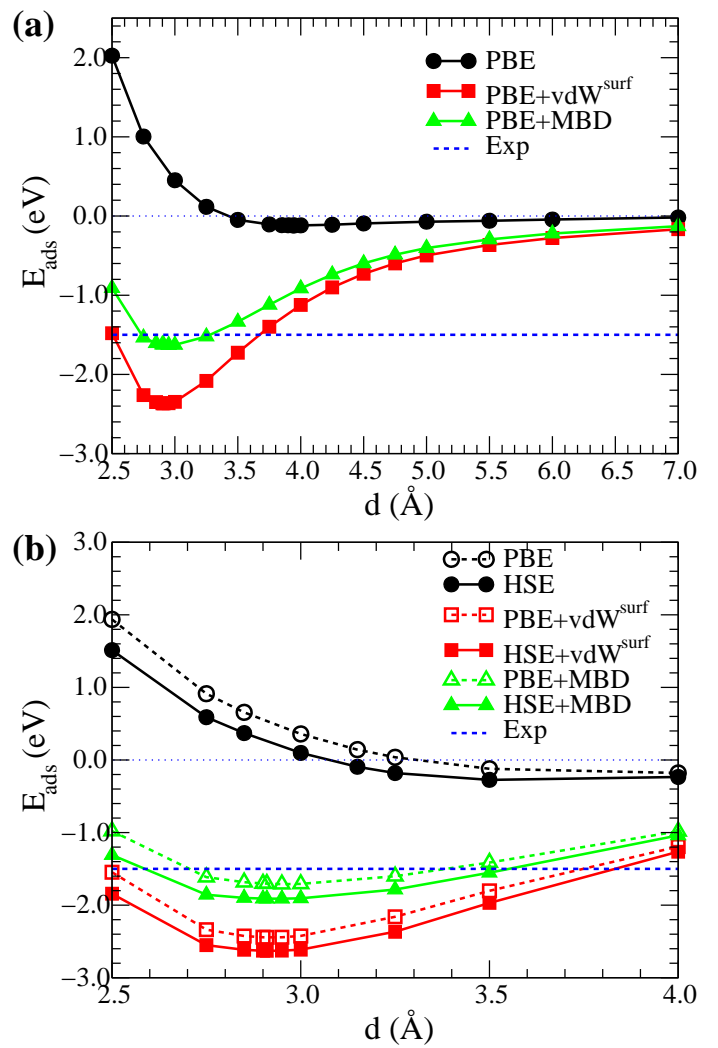

FIG. 7. Adsorption energy $E_{\text {ads }}$ of bridge60pentacene $/ \operatorname{Ag}(111)$ as a function of the adsorption height $d$, computed using the (a) PBE, PBE+vdW ${ }^{\text {surf }}$ and $\mathrm{PBE}+\mathrm{MBD}$, and (b) HSE, HSE+vdW ${ }^{\text {surf }}$ and HSE+MBD approaches. The geometry was constrained to the PBE equilibrium geometry in the $\mathrm{PBE} / \mathrm{HSE}$ calculations and

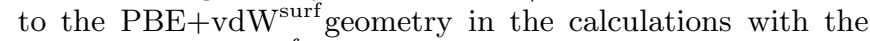
$\mathrm{PBE} / \mathrm{HSE}+\mathrm{vdW}^{\text {surf }}$ and $\mathrm{PBE} / \mathrm{HSE}+\mathrm{MBD}$ methods, and only the adsorption height was changed. The blue dashed lines represent the experimental value of the desorption energy of pentacene/ $\mathrm{Ag}(111)$ reported in Ref. 9.

(Fig. 8(c)) calculations predicted that the peak of the LUMO is at $\sim 0.06$ and $0.07 \mathrm{eV}$ above the Fermi level, respectively. Although we expect the LUMO position be underestimated since Kohn-Sham levels are unsuitable to describe excited states, this finding suggests that the interaction between molecule and substrate is stronger in pentacene $/ \mathrm{Ag}(111)$. As we will discuss later, this will be reflected in the change of the work function of $\mathrm{Ag}(111)$ upon adsorption of pentacene and anthracene; pentacene induces a smaller reduction in the work function than anthracene. Regarding the occupied states, the HSE functional (Fig. 8(c)) predicted that the HOMO is located at $\sim 1.38 \mathrm{eV}$ below the Fermi level, which is in good agreement with the binding energy of $1.5 \mathrm{eV}$ obtained by ultraviolet photoelectron spectroscopy ${ }^{8}$. Our PBE calculations overestimated the position of the HOMO state, placing it at about $1.0 \mathrm{eV}$ below the Fermi level (Fig. 8(b)).

Comparing Figs. 8(b) and (d) we notice that 
(a)

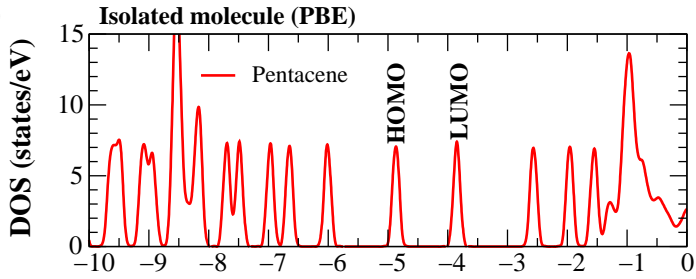

(b)

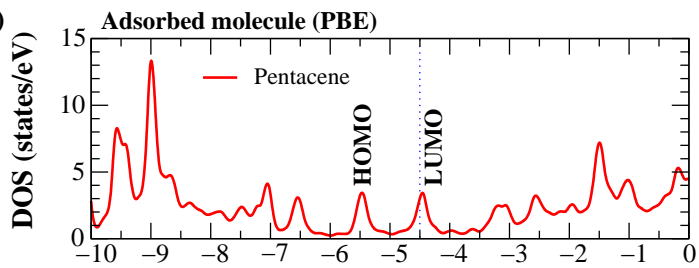

(c)

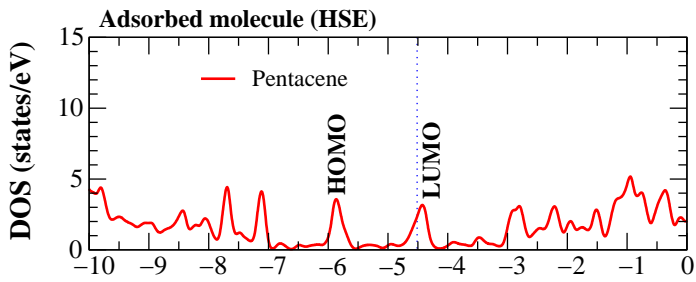

(d)

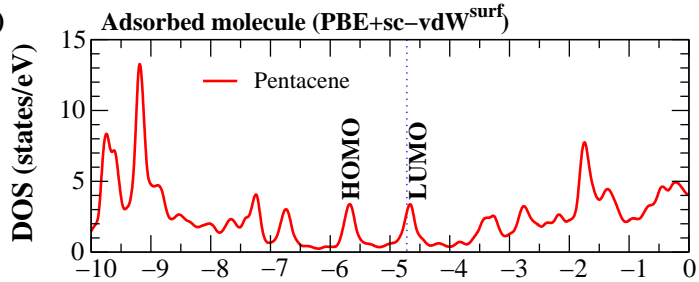

(e)

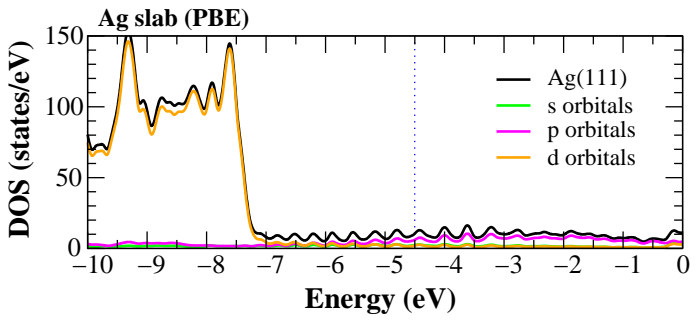

FIG. 8. Calculated density of states (DOS) of the pentacene $/ \mathrm{Ag}(111)$ system in the optimized PBE + vdW ${ }^{\text {surf }}$ geometry. (a) Isolated pentacene, (b-d) density of states projected onto the molecular orbitals of pentacene/ $\operatorname{Ag}(111)$ obtained with (b) PBE and (c) HSE functionals and (d) the $\mathrm{PBE}+\mathrm{sc}-\mathrm{vdW} \mathrm{Wrr}^{\text {surf }}$ method, and (e) clean $\operatorname{Ag}(111)$ surface. The zero on the energy scale corresponds to the vacuum level. The dashed lines indicate the position of the Fermi level.

the inclusion of the vdW interactions using the sc$\mathrm{vdW}^{\text {surf }}$ method does not alter the electronic properties of pentacene $/ \mathrm{Ag}(111)$ by more than a constant energy shift (see also Fig. S6 of the Supplemental Material). Similar to what was observed for anthracene/ $\operatorname{Ag}(111)$, vdW interactions are important for the description of the stability and geometry of the system, but have no significant effect on its electronic structure once the correct geometry has been obtained. Again, we found that the effect of the vdW interactions on the elec- tronic structure of pentacene/ $\operatorname{Ag}(111)$ is indirect, via the correct description of the geometry of the system; as can be seen in Fig. S4(a), the DOS of the adsorbed molecule at the PBE geometry is similar to the DOS of the isolated molecule (Fig. 8(a)), whereas the DOS of the adsorbed molecule at the $\mathrm{PBE}+\mathrm{vdW} \mathrm{W}^{\text {surf }}$ geometry (Fig. 8(b)) is signiticantly different, with broader HOMO and LUMO peaks and the LUMO state located closer to the Fermi level (see also Fig. S4 for a comparison between the DOS of the adsorbed molecule at the PBE

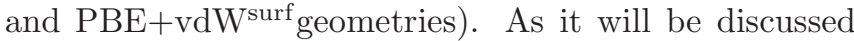
in the next paragraph, the proximity of the LUMO state to the Fermi level strongly affects the change in the work function of the $\operatorname{Ag}(111)$ surface due to the adsorption of pentacene molecules.

Lastly, we also computed the change in the work function of the $\operatorname{Ag}(111)$ surface due to the adsorption of a pentacene monolayer. Using "pure" PBE calculations we found that the work function decreases from $4.43 \mathrm{eV}$ to $3.98 \mathrm{eV}$, a reduction of $0.45 \mathrm{eV}$; with the $\mathrm{PBE}+\mathrm{sc}-$ vdW ${ }^{\text {surf }}$ method, we found that the work function decreases by $0.73 \mathrm{eV}$, changing from 4.81 to $4.08 \mathrm{eV}$. Our results for the work function of the pentacene/ $\mathrm{Ag}(111)$ system $(\phi=3.98$ and $4.08 \mathrm{eV})$, as well as the computed reduction $(\Delta \phi=-0.45$ and $-0.73 \mathrm{eV})$ are in close agreement with experimental values reported in Ref. $8, \phi=3.95 \mathrm{eV}$ and $\Delta \phi=-0.5 \mathrm{eV}$, and in Ref. 45, $\phi=4.00 \mathrm{eV}$ and $\Delta \phi=-0.59 \mathrm{eV}$. We noticed, in addition, that the change in the work function of $\mathrm{Ag}(111)$ induced by the adsorption of the pentacene monolayer is substantially smaller than that caused by the adsorption of anthracene. In order to explain this finding, one can think of the work function change as being composed of two opposing components: the first one is the pushback effect, which dominates in anthracene/ $\operatorname{Ag}(111)$ and is present in pentacene/ $\mathrm{Ag}(111)$ as well; the second contribution originates from an accumulation of charge on the molecule and/or in the space between the molecule and the surface, which tends to increase the work function and thus counteracts the push-back effect. This latter effect, while being absent for anthracene, starts to play some role for pentacene and larger oligoacenes. On the level of our DFT calculations this can be concluded from the hybridization with the substrate states and energetic broadening of the LUMO seen in Fig. 8. Even if the very low energetic position of the LUMO may be an artefact of the DFT calculations, we think that the trends observed for the change in work function will persist in a higher-level treatment of the electronic structure: also in post-Hartree-Fock methods, we expect induced fractional charges on the adsorbed molecule to counteract the original work function lowering due to the pushback effect. The size of possibly induced charges scales with the polarisability of the free molecule. The larger the oligoacenes, the more polarisable they are, hence the trend to smaller work function change when larger molecules are adsorbed. 


\section{CONCLUSIONS}

In summary, we investigated the effects of the vdW interactions on the structural and electronic properties of anthracene $/ \operatorname{Ag}(111)$ and pentacene $/ \mathrm{Ag}(111)$. We employed two methods to treat the $\mathrm{vdW}$ interactions in our calculations: the vdW ${ }^{\text {surf }}$ approach and the MBD method. Our results show that the inclusion of $\mathrm{vdW}$ corrections is crucial to correctly describe the binding geometries of these systems. For anthracene/ $\mathrm{Ag}(111)$, in particular, "pure" PBE calculations favored a tilted configuration whereas the $\mathrm{PBE}+\mathrm{vdW} \mathrm{Wurf}^{\text {supproach yielded }}$ a flat-lying geometry, in agreement with previous experimental studies. We found, additionally, that $\mathrm{vdW}$ interactions strongly affect the adsorption energies and heights of anthracene/ $\operatorname{Ag}(111)$ and pentacene $/ \operatorname{Ag}(111)$; both $\mathrm{PBE}+\mathrm{vdW} \mathrm{W}^{\text {surf }}$ and $\mathrm{PBE}+\mathrm{MBD}$ methods predicted adsorption heights considerably smaller (between 2.9 and $3.1 \AA$, while PBE results are larger than $3.9 \AA$ ) and adsorption energies significantly larger (more than one order of magnitude larger) than the PBE results, in better agreement with experimental data and more consistent with previous studies on other oligoacenes adsorbed on the $\operatorname{Ag}(111)$ surface. We also examined the effect of the vdW interactions on the electronic properties of anthracene $/ \operatorname{Ag}(111)$ and pentacene $/ \operatorname{Ag}(111)$, by using the self-consistent $\mathrm{vdW}^{\text {surf }}$ method. We found that the inclusion of the self-consistency of the vdW energy in our calculations has only tiny effects on the electronic structure of these systems, indicating that the major influence of the vdW interactions is in the stability and structural properties of organic/metal systems.

Analyses of the density of states of anthracene $/ \operatorname{Ag}(111)$ and pentacene $/ \operatorname{Ag}(111)$ obtained using the PBE, HSE and $\mathrm{PBE}+\mathrm{sc}-\mathrm{vdW}$ surf methods revealed that the LUMO level of an anthracene monolayer remains unoccupied upon adsorption on $\mathrm{Ag}(111)$ indicating physisorption for anthracene/ $\mathrm{Ag}(111)$; in pentacene/ $\mathrm{Ag}(111)$ the LUMO peak is located right above the Fermi level, suggesting stronger interaction between molecule and substrate. The stronger molecule-metal interaction in pentacene $/ \mathrm{Ag}(111)$ explains the smaller reduction in the work function of the $\mathrm{Ag}(111)$ surface obtained upon pentacene adsorption (PBE: $-0.45 \mathrm{eV}$, PBE+sc-vdW ${ }^{\text {surf }}$ : $-0.73 \mathrm{eV}$ ) when compared to that computed for the adsorption of an anthracene monolayer (PBE: $-0.81 \mathrm{eV}, \mathrm{PBE}+\mathrm{sc}-\mathrm{vdW} \mathrm{W}^{\text {surf }}$ : $-1.04 \mathrm{eV}$ ).

\section{SUPPLEMENTARY MATERIAL}

See supplementary material for further details on the density of states of anthracene/ $\mathrm{Ag}(111)$ and pentacene/Ag(111).

\section{ACKNOWLEDGMENTS}

We acknowledge fruitful discussions within the collaborative research center SFB 1242 "Non-equilibrium dynamics in condensed matter in the time domain" funded by the Deutsche Forschungsgemeinschaft. The authors gratefully acknowledge the computing time granted by the Center for Computational Sciences and Simulation (CCSS) of the University of Duisburg-Essen and provided on the supercomputer magnitUDE (DFG grant INST 20876/209-1 FUGG) at the Zentrum für Informationsund Mediendienste (ZIM).

${ }^{1}$ S. F. Nelson, Y.-Y. Lin, D. J. Gundlach, and T. N. Jackson, Applied Physics Letters 72, 1854 (1998); O. D. Jurchescu, J. Baas, and T. T. M. Palstra, ibid. 84, 3061 (2004).

${ }^{2}$ S. Tao, S. Xu, and X. Zhang, Chemical Physics Letters 429, 622 (2006).

${ }^{3}$ M. Kitamura, T. Imada, and Y. Arakawa, Applied Physics Letters 83, 3410 (2003); T. Dobbertin, M. Kroeger, D. Heithecker, D. Schneider, D. Metzdorf, H. Neuner, E. Becker, H.-H. Johannes, and W. Kowalsky, ibid. 82, 284 (2003).

${ }^{4}$ V. Y. Butko, X. Chi, D. V. Lang, and A. P. Ramirez, Applied Physics Letters 83, 4773 (2003); C. D. Dimitrakopoulos, S. Purushothaman, J. Kymissis, A. Callegari, and J. M. Shaw, Science 283, 822 (1999).

${ }^{5}$ P. Yannoulis, K.-H. Frank, and E.-E. Koch, Surface Science 241, 325 (1991).

${ }^{6}$ T. Shimooka, S. Yoshimoto, M. Wakisaka, J. Inukai, and K. Itaya, Langmuir 17, 6380 (2001).

${ }^{7}$ K. H. Frank, P. Yannoulis, R. Dudde, and E. E. Koch, The Journal of Chemical Physics 89, 7569 (1988).

${ }^{8}$ N. Koch, I. Salzmann, R. Johnson, J. Pflaum, R. Friedlein, and J. Rabe, Organic Electronics 7, 537 (2006).

${ }^{9}$ D. B. Dougherty, W. Jin, W. G. Cullen, J. E. Reutt-Robey, and S. W. Robey, The Journal of Physical Chemistry C 112, 20334 (2008).

${ }^{10}$ S. Duhm, C. Bürker, J. Niederhausen, I. Salzmann, T. Hosokai, J. Duvernay, S. Kera, F. Schreiber, N. Koch, N. Ueno, and A. Gerlach, ACS Applied Materials \& Interfaces 5, 9377 (2013).

${ }^{11}$ A. Tkatchenko, L. Romaner, O. T. Hofmann, E. Zojer, C. Ambrosch-Draxl, and M. Scheffler, MRS Bulletin 35, 435 (2010).

${ }^{12}$ G. Li, I. Tamblyn, V. R. Cooper, H.-J. Gao, and J. B. Neaton, Phys. Rev. B 85, 121409 (2012).

${ }^{13}$ W. Liu, A. Tkatchenko, and M. Scheffler, Accounts of Chemical Research 47, 3369 (2014).

${ }^{14}$ W. Liu, J. Carrasco, B. Santra, A. Michaelides, M. Scheffler, and A. Tkatchenko, Phys. Rev. B 86, 245405 (2012).

${ }^{15}$ M. Dion, H. Rydberg, E. Schröder, D. C. Langreth, and B. I. Lundqvist, Phys. Rev. Lett. 92, 246401 (2004).

${ }^{16}$ D. C. Langreth, B. I. Lundqvist, S. D. Chakarova-Käck, V. R. Cooper, M. Dion, P. Hyldgaard, A. Kelkkanen, J. Kleis, L. Kong, S. Li, P. G. Moses, E. Murray, A. Puzder, H. Rydberg, E. Schröder, and T. Thonhauser, Journal of Physics: Condensed Matter 21, 084203 (2009).

${ }^{17}$ S. Grimme, Journal of Computational Chemistry 27, 1787 (2006).

18 A. Tkatchenko and M. Scheffler, Phys. Rev. Lett. 102, 073005 (2009).

${ }^{19}$ V. G. Ruiz, W. Liu, E. Zojer, M. Scheffler, and A. Tkatchenko, Phys. Rev. Lett. 108, 146103 (2012).

${ }^{20}$ A. Tkatchenko, R. A. DiStasio, R. Car, and M. Scheffler, Phys. Rev. Lett. 108, 236402 (2012).

${ }^{21}$ A. Ambrosetti, A. M. Reilly, R. A. DiStasio, and A. Tkatchenko, J. Chem. Phys. 140, 18A508 (2014).

${ }^{22}$ R. J. Maurer, V. G. Ruiz, and A. Tkatchenko, The Journal of Chemical Physics 143, 102808 (2015). 
${ }^{23}$ V. G. Ruiz, W. Liu, and A. Tkatchenko, Phys. Rev. B 93, 035118 (2016).

${ }^{24}$ J. Carrasco, W. Liu, A. Michaelides, and A. Tkatchenko, The Journal of Chemical Physics 140, 084704 (2014).

${ }^{25}$ W. Kohn and L. J. Sham, Phys. Rev. 140, A1133 (1965).

${ }^{26}$ V. Blum, R. Gehrke, F. Hanke, P. Havu, V. Havu, X. Ren, K. Reuter, and M. Scheffler, Comput. Phys. Commun. 180, 2175 (2009).

${ }^{27}$ J. P. Perdew, K. Burke, and M. Ernzerhof, Phys. Rev. Lett. 77, 3865 (1996).

${ }^{28}$ J. Heyd, G. E. Scuseria, and M. Ernzerhof, J. Chem. Phys. 118, 8207 (2003); 124, 219906 (2006).

${ }^{29}$ E. Lifshitz, Sov. Phys. JETP , 73 (1956).

${ }^{30}$ E. Zaremba and W. Kohn, Phys. Rev. B 13, 2270 (1976).

${ }^{31}$ N. Ferri, R. A. DiStasio, A. Ambrosetti, R. Car, and A. Tkatchenko, Phys. Rev. Lett. 114, 176802 (2015).

${ }^{32}$ V. L. Moruzzi, J. F. Janak, and K. Schwarz, Phys. Rev. B 37, 790 (1988).

${ }^{33}$ W. Liu, V. G. Ruiz, G.-X. Zhang, B. Santra, X. Ren, M. Scheffler, and A. Tkatchenko, New Journal of Physics 15, 053046 (2013).

${ }^{34}$ A. Hauschild, K. Karki, B. C. C. Cowie, M. Rohlfing, F. S. Tautz, and M. Sokolowski, Phys. Rev. Lett. 94, 036106 (2005).

${ }^{35}$ L. Kilian, W. Weigand, E. Umbach, A. Langner, M. Sokolowski, H. L. Meyerheim, H. Maltor, B. C. C. Cowie, T. Lee, and P. Bäuerle, Phys. Rev. B 66, 075412 (2002).

${ }^{36}$ W. Liu, F. Maaß, M. Willenbockel, C. Bronner, M. Schulze, S. Soubatch, F. S. Tautz, P. Tegeder, and A. Tkatchenko, Phys.
Rev. Lett. 115, 036104 (2015).

${ }^{37}$ R. J. Maurer, V. G. Ruiz, J. Camarillo-Cisneros, W. Liu, N. Ferri, K. Reuter, and A. Tkatchenko, Progress in Surface Science 91, $72(2016)$.

${ }^{38}$ T. Rockey and H.-L. Dai, Surface Science 601, 2307 (2007).

${ }^{39}$ X. Blase, C. Attaccalite, and V. Olevano, Phys. Rev. B 83, 115103 (2011).

${ }^{40}$ L. Romaner, D. Nabok, P. Puschnig, E. Zojer, and C. AmbroschDraxl, New Journal of Physics 11, 053010 (2009).

${ }^{41}$ H. B. Michaelson, Journal of Applied Physics 48, 4729 (1977).

${ }^{42}$ S. Duhm, A. Gerlach, I. Salzmann, B. Bröker, R. Johnson, F. Schreiber, and N. Koch, Organic Electronics 9, 111 (2008).

${ }^{43}$ Y. Zou, L. Kilian, A. Schöll, T. Schmidt, R. Fink, and E. Umbach, Surface Science 600, 1240 (2006).

${ }^{44}$ K. J. Gaffney, A. D. Miller, S. H. Liu, , and C. B. Harris, The Journal of Physical Chemistry B 105, 9031 (2001).

${ }^{45}$ M.-C. Lu, R.-B. Wang, A. Yang, and S. Duhm, Journal of Physics: Condensed Matter 28, 094005 (2016).

${ }^{46}$ M. Eremtchenko, R. Temirov, D. Bauer, J. A. Schaefer, and F. S. Tautz, Phys. Rev. B 72, 115430 (2005).

${ }^{47}$ K. Toyoda, I. Hamada, K. Lee, S. Yanagisawa, and Y. Morikawa, The Journal of Chemical Physics 132 (2010), http://dx.doi.org/10.1063/1.3373389.

${ }^{48}$ E. Mete, İlker Demiroğlu, M. F. Danışman, and Şinasi Ellialtıŏlu, The Journal of Physical Chemistry C 114, 2724 (2010).

${ }^{49}$ J. Björk and S. Stafström, Chem. Phys. Chem. 15, 2851 (2014). 